\title{
ASOCIACIÓN DE DATOS ESPACIO-TEMPORALES EN BASES DE DATOS ORACLE
}

\author{
Claudia Elena Durango Vanegas \\ Universidad de San Buenaventura seccional Medellín \\ claudia.durango@usbmed.edu.co
}

(Tipo de Artículo: Reflexión. Recibido el 06/06/2014. Aprobado el 26/12/2014)

\begin{abstract}
RESUMEN
Los Sistemas de Información Geográfica (SIG) comprenden un conjunto de herramientas para capturar, almacenar, recuperar, transformar y visualizar información espacial del mundo real. El tiempo es una dimensión para modelar la evolución de comportamientos geográficos. Los formatos de datos espacio-temporales como XML, KML y GML se utilizan para almacenar este tipo de comportamientos geográficos en datos vector. La estructura de los datos SIG actualmente son estáticas, no pueden manejar la dinámica y la interacción de los objetos de un entorno 3D dinámico. Esto significa que la integración de los datos basados en el tiempo en SIG para la simulación es compleja. Algunos métodos de almacenamiento discreto de los datos en bases de datos se basan en describir las variaciones del tiempo en secuencias de eventos y del tiempo, pero existen problemas para su uso por no contar con una estructura unificada de almacenamiento en los sistemas de gestión de datos geográfico que soporten algunos formatos espacio-temporales, lo que puede generar problemas y falta de eficiencia en la difusión y reutilización de la información. Por lo anterior, en este artículo se propone un modelo de gestión de datos geográficos en Oracle para la representación de los formatos espacio-temporales XML, GML y KML. En el modelo se observan las tablas y relaciones de los elementos principales de dichos formatos espacio-temporales.
\end{abstract}

Palabras clave. Datos Espacio-Temporales, Esquemas Preconceptuales, Sistemas de Información Geográfica, Oracle.

\section{ASSOCIATION OF SPATIO-TEMPORAL DATA IN ORACLE DATABASES}

\begin{abstract}
Geographic Information Systems (GIS) covers a set of tools for capturing, storing, retrieving, transforming and displaying spatial data from real world. Time is a dimension to model the evolution of geographic behavior. The spatio-temporal data formats like $X M L, K M L$ and GML are used to store this type of behavior in geographic vectors data. The structure of GIS data currently is static; it cannot manage the dynamics and object interaction of a 3D dynamic environment which means that the integration of time-based data in GIS for simulation is complex. Some methods of discrete data storage in databases are based on describing time variations in sequences of events and time. However, some troubles were found because they do not have a unified storage structure in database management systems that support some geographic spatio-temporal formats, this could result in problems and inefficiency when distributing and reusing information. Therefore, this paper proposes a model for managing geographic data in Oracle to represent spatio-temporal formats such as XML, GML and KML. The model has tables and relations of the main elements of these formats.
\end{abstract}

Keywords. Spatio-Temporal Data, Pre-conceptual Schemes, Geographic Information Systems, Oracle

\section{Association de données spatio-temporelles sur bases de données Oracle}

\begin{abstract}
Résumé
Les systèmes d'information géographique (SIG) comprennent un ensemble d'outils pour capturer, stocker, transformer et visualiser information spatiale du monde réel. Le temps est une dimension pour modeler l'évolution des comportements géographiques. Les formats des donnes spatio-temporelles comme XML, KML et GML sont utilisés pour stocker ce type de comportements géographiques en données vecteur. La structure des données SIG sont statiques dans l'actualité, elles ne peuvent pas régler la dynamique et l'interaction des objets d'un environnement $3 D$ dynamique. Ça signifie que l'intégration des données en se basant sur le temps dans SIG pour la simulation est complexe. Quelques méthodes de stockage discret des données sur bases de données se basent sur la description des variations du temps dans séquences d'événements et du temps, mais il y a des problèmes pour son usage parce qu'ils n'ont pas une structure unifié de stockage dans les systèmes de gestion de données géographiques qui supportent quelques formats spatio-temporelles, ce qui peut produire des problèmes et manque d'efficacité dans la diffusion et réutilisation de l'information. Par conséquent, on propose un modèle de gestion de données géographiques sur Oracle pour représenter les formats spatio-temporelles XML, GML et KML. Dans le model on trouve les tables et relations des éléments principaux de tels formats.
\end{abstract}

Mots-clés. Données spatio-temporelles, schémas pre-conceptuels, systèmes d'information géographique, Oracle. 


\section{INTRODUCCIÓN}

Los Sistemas de Información Geográfica (SIG) comprenden un conjunto de herramientas para capturar, almacenar, recuperar, transformar y visualizar información espacial del mundo real. Uno de los elementos importantes en el diseño de los SIG es la forma de estructurar los datos geográficos en los sistemas de gestión de bases de datos para realizar análisis espaciales. Los datos geográficos contienen elementos tabulares y tienen asociados elementos geográficos como localización, sistema de coordenadas, extensión y relaciones geográficas [1]. Los datos espacio-temporales representan el espacio y el tiempo. Las coordenadas espaciales y el tiempo están mutuamente relacionados, un cambio en un atributo (espacial o no espacial) en el tiempo implica la introducción de un dato histórico a la base de datos [2]. La importancia de las bases de datos espaciales es que permiten almacenar información relativa a un conjunto de entidades espaciales (geometría, topología, identificadores, datos temáticos, entre otros). En este artículo se propone incorporar los atributos mínimos de los formatos de datos espacio-temporales para datos vector en un sistema de gestión de bases de datos en Oracle.

El artículo se organiza de la siguiente manera: en la Sección 2 se presenta el marco teórico, donde se realiza una descripción de los Sistemas de Información Geográfica, los datos espacio-temporales, los Esquemas Preconceptuales y las bases de datos espaciales; en la Sección 3 se presentan los antecedentes, donde se revisan los diferentes sistemas de gestión de bases de datos en SIG; en la Sección 4 se presentan los elementos principales de los formatos de datos espacio-temporales utilizando Esquemas Preconceptuales y una propuesta para la incorporación de datos espacio-temporales para datos vector en bases de datos Oracle. Finalmente, en la Sección 6 se presentan las conclusiones y trabajos futuros.

\section{MARCO CONCEPTUAL}

Los Sistemas de Información Geográfica (SIG) son aplicaciones que poseen datos relacionados con coordenadas geográficas (datos espaciales) y atributos descriptivos [3]. Los datos espaciales o geodatos se refieren a localizaciones específicas en el espacio. Los sistemas de coordenadas son la fuente principal para determinar la ubicación de un objeto o fenómeno en la superficie de la tierra. El análisis de geodatos está relacionado con la localización en el espacio geográfico, en otras palabras localización sobre o cerca de la superficie de la tierra [4]. Los geodatos describen ciudades, vías, afluentes, predios, entre otros datos con ubicación espacial. Los geodatos tienen asociados datos espaciales y atributos descriptivos (véase la Figura 1). Los atributos descriptivos están relacionados con la información tipo textos y valores asociados a los datos espaciales [1] (véase la Figura 2) [5].

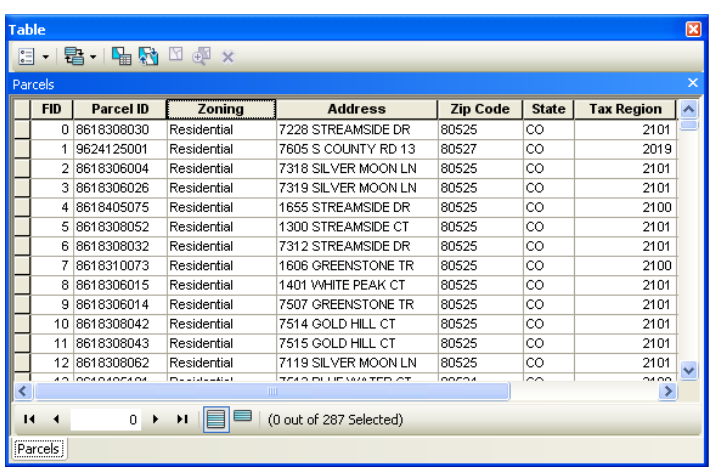

Figura 1. Representación de atributos descriptivos [1]

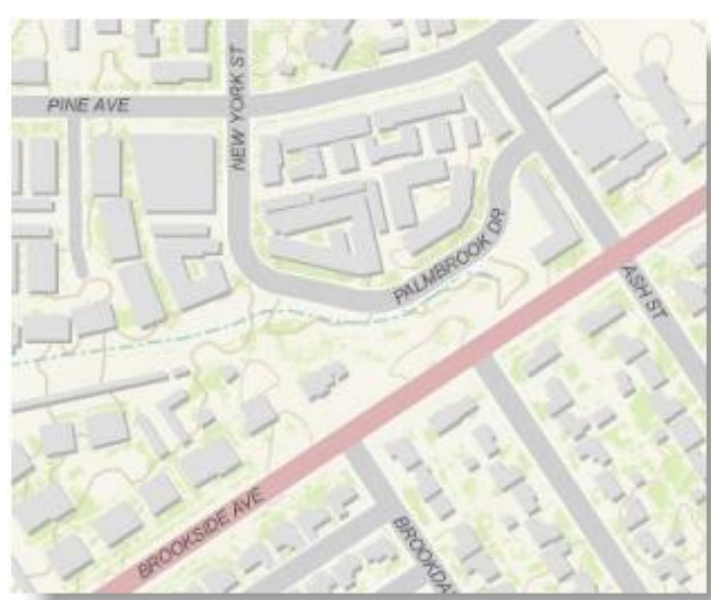

Figura 2.Representación de datos espaciales [1]

Las estructuras básicas de almacenamiento de un SIG son vector y ráster. Los datos vector son puntos, líneas y polígonos con capacidad de almacenar diferentes datos descriptivos (véase la Figura 3;Error! No se encuentra el origen de la referencia.). Los datos ráster son pixeles o puntos que representan imágenes y tienen asociado un solo dato descriptivo numérico como: temperatura, altura, condiciones climáticas, entre otras (véase la Figura 4) [2].

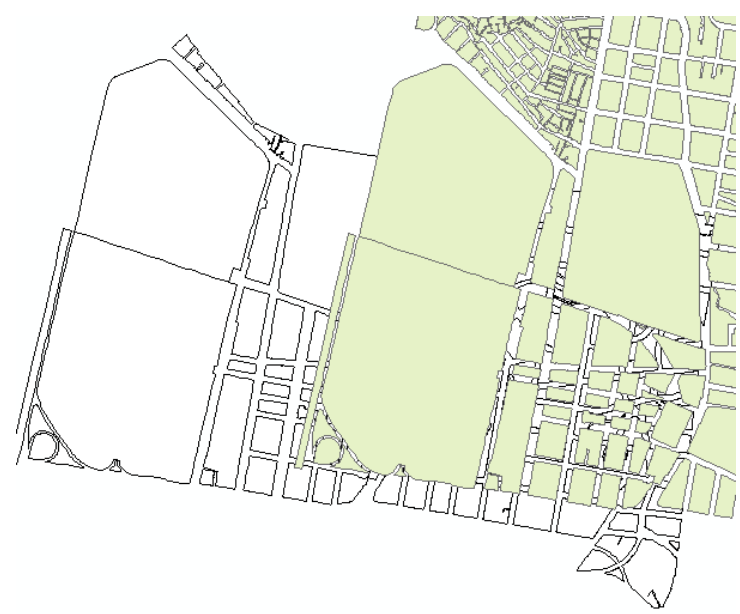

Figura 3. Visualización de datos vector (polígono) en un SIG. Construcción propia empleando el SIG ArcGis $\mathbf{1 0 . 2}$ 


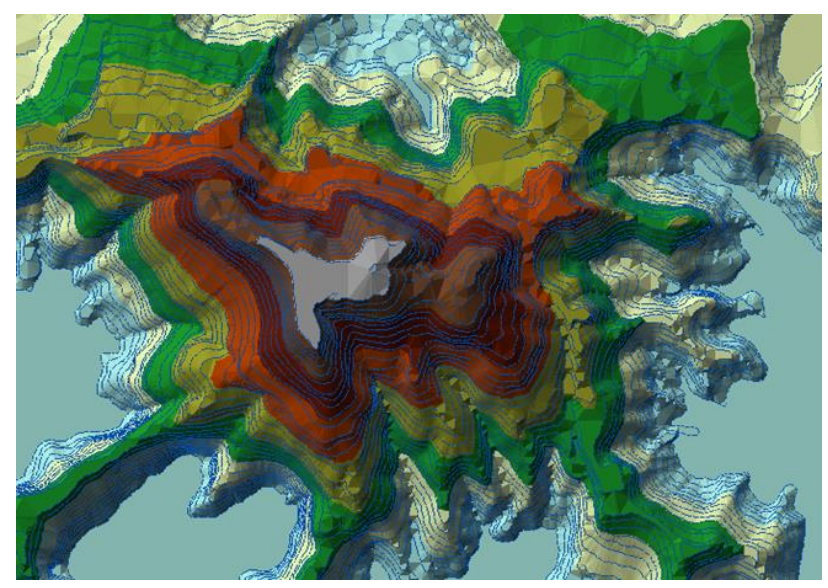

Figura 4. Visualización de datos ráster en un SIG. Construcción propia empleando el SIG ArcGis $\mathbf{1 0 . 2}$

Los datos espaciales se representan principalmente por la ubicación geográfica mediante latitud y longitud, coordenada $\mathrm{X}$ y coordenada $\mathrm{Y}$, en general en dos dimensiones georreferenciadas. Los fenómenos y actividades espacio-temporales dependen directamente de la transformación del espacio geográfico en un determinado periodo de tiempo [7]. Diversos estudios examinan el espacio y el tiempo, asumiendo que cada elemento geográfico desempeña múltiples roles en un momento específico del tiempo; esto conduce a sugerir que no existe un área en el espacio geográfico que se pueda separar del flujo del tiempo y el espacio. Las entidades geográficas presentan una ruta espacio-temporal (véase la Figura 5), que inicia en el momento de la toma del geodato y termina en el momento que se destruyen el geodato. Bajo la anterior descripción, los datos espaciotemporales se representan como una inserción de la dimensión tiempo en entidades geográficas concebidas, donde el espacio geográfico se organiza en capas temáticas que incluyen la información de captura en un tiempo determinado [8].

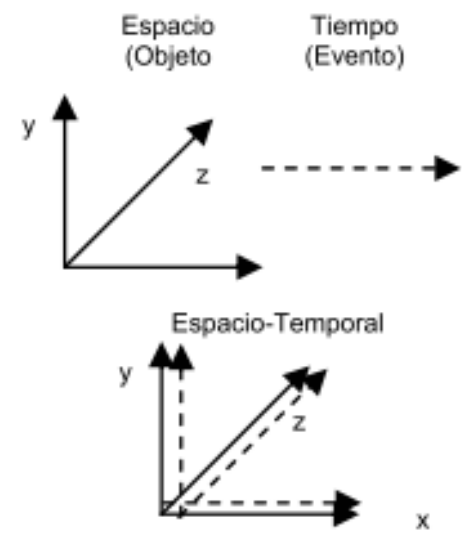

Figura 5. Representación Entidad Espacio-Temporal [3]

Los Esquemas Preconceptuales son una representación de un dominio especifico y emplean simbología propia (véase Figura 6), se conciben como una especificación semiformal que cualquier persona puede usar y entender. Los Esquemas Preconceptuales facilitan la comunicación entre personas con conocimientos técnicos de modelado y los conocedores del dominio que se está modelando [4].

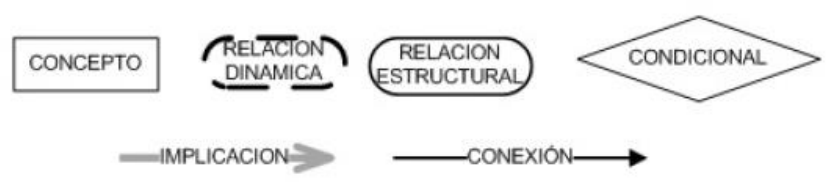

Figura 6. Simbología Básica de los Esquemas Preconceptuales [2]

Los eventos relacionados con la geoinformación describen aspectos temporal, posición en el tiempo, extensión temporal que denotan un cambio de comportamiento [5]. Debido a la amplia utilización de la geoinformación surge Open Geospacial Consortium (OGC). OGC es una organización internacional sin ánimo de lucro que lidera el desarrollo de estándares para servicios geoespaciales [6]. Los principales formatos de datos espacio-temporales para datos vector son:

- Extensible Markup Language (XML) es un formato de texto simple, muy flexible derivado de SGML (ISO 8879). Originalmente, se diseñó para afrontar los retos de la edición electrónica.

- Geography Markup Language (GML) es un estándar de codificación basado en XML para información geográfica, que desarrolló el OpenGIS Consortium [6], [7]. GML sirve para modelar, transportar y almacenar información geográfica, relacionada con la posición, localización y extensión, entre otros; incluye propiedades espaciales y no espaciales de las entidades geográficas[6].

- Keyhole Markup Language (KML) es un lenguaje de marcado descriptivo, basado en la sintaxis del formato XML, para representar datos geográficos en tres dimensiones. KML permite describir y almacenar información geográfica (puntos, líneas, superficies, polígonos) y utiliza como referencia el estándar GML. KML permite la representación de relaciones topológicas en modelos geográficos, optimiza la descripción de elemento y describe la información geográfica mediante etiquetas con elementos anidados [8].

Un Sistema Gestor de Bases de Datos (SGBD) es un software que permite la definición de las bases de datos, permitiendo la elección de la estructura de los datos requeridos para el almacenamiento y búsqueda de datos. Para los SIG se utiliza una extensión del SGBD incorporando datos espaciales en el modelo de datos y en sus consultas [9]. Existen principalmente dos tipos de sistema de gestión de datos geográficos: Spatial Database y Temporal Database. Una Spatial Database representa una base de datos que optimiza el almacenamiento y consulta de datos relacionados a objetos en el espacio (puntos, líneas y polígonos) y 
operaciones espaciales como intercepción y distancia. Una Temporal Geodatabase permite almacenar datos espaciales asociados a un periodo de tiempo. Existen dos notaciones del tiempo en una Temporal Geodatabase: tiempo valido y tiempo de transacción. EI tiempo válido es el periodo de tiempo durante el cual un hecho es verdadero con respecto al mundo real. El tiempo de transacción es el periodo de tiempo durante el cual un hecho se almacena en la base de datos espacial. Los dos periodos de tiempo no tienen que ser los mismos para un hecho [10].

\section{ANTECEDENTES}

Erwing et ál. (1998) explican los componentes de base de datos espacial como un conjunto de clases de objetos de diferentes tipos y esquemas. Cada clases tiene asociado objetos, cada objeto asocia un número de atributos con elementos espacial (dibujo), dominio y tipo de dato. Además, la base de datos espacial contiene features como objetos, valores de los atributos, métodos, clases de objetos y relaciones, entre otros. El modelo abstracto y discreto de los tipos de datos espacio-temporales tiene en cuenta las transacciones del tiempo en las bases de datos, donde los cambios geométricos pasan solo en datos discretos al actualizar la base de datos. El artículo describe y discute una aproximación del modelo de objetos espaciales en movimiento específicamente para puntos y áreas con operaciones y entidades. Se considera un modelo básico relacionado con el momento de crear o destruir un objeto espacial, asociando a un intervalo de validez [11]. Los autores proponen un modelo de tipos de datos para el describir los cambios en el espacio y el tiempo, pero no muestran un modelo entidad-relación que explique el comportamiento de las clases, objetos y atributos en la base de datos espacial.

Li y Cai (2002) identificaron que al modelar datos espaciales los aspectos temporales son modelados separadamente y encontraron problemas para representar fenómenos continuos, donde muchos sistemas temporales capturar datos discretos (dato vector) y no tienen en cuenta los datos continuos (dato ráster). El modelo tiene una estructura de las clases compuesta por la súper clase, la estructura de los atributos y el comportamiento. La súper clase está relacionado por las subclases en diferentes contextos de aplicación (véase Figura 7). La estructura de los atributos está relacionada con que cada objeto tiene atributos de tema, espacio y tiempo. Los atributos del tema están relacionados con ¿Qué es el objeto? y otras propiedades. Los atributos del espacio guardan la información geométrica del objeto; punto, línea o polígono, y su localización. Los atributos del tiempo representan cambios en el tiempo asociados al tema o geometría. Cuando la información de las propiedades o geometría cambia, un nuevo cambio en el tiempo se asocia al objeto. Lo que significa que solo la información inicial y final se guarda. En el modelo la información temática, espacial y del tiempo se guarda en tres tablas con sus respectivas relaciones (uno-uno, uno a muchos y mucho a muchos) (véase Figura 8) [12]. Los autores proponen un modelo de datos espacio temporal orientado a objetos que tiene en cuenta los atributos básicos y el comportamiento de los objetos espacio-temporales.

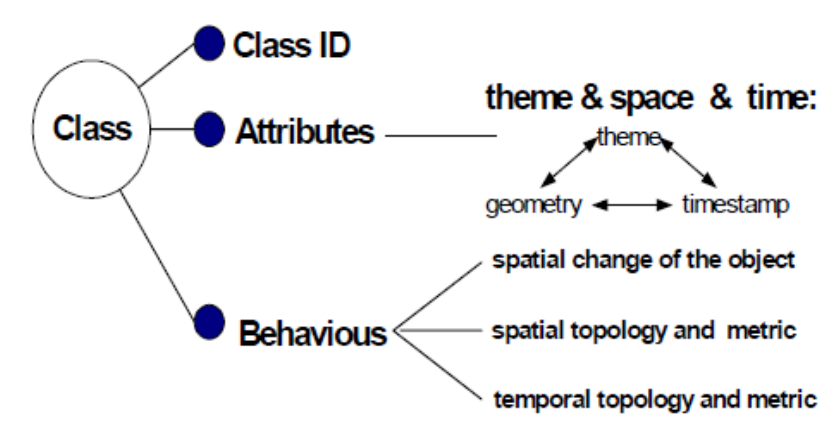

Figura 7. Estructura de la súper clase

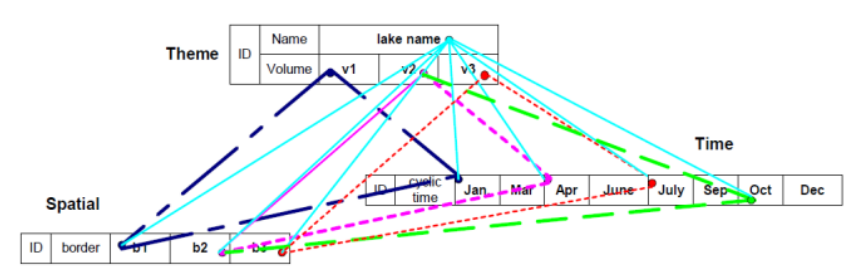

Figura 8. Relaciones entre temas, espacio y tiempo

Li et ál. (2004) proponen una arquitectura para almacenar archivos GML en una base de datos espacial en Oracle. La arquitectura consiste en cuatro principales componentes: Schema Tree Generatos (STG), Mapping Rules Generatos (MRG), Data Loader (DL) y Query Translator (QT). Los autores almacenaron los atributos de la información geográfica en tablas relacionales y la información espacial se asigna en el archivo GML a la columna Geometry con la geometría del dato. Oracle Spatial Database tiene una columna especial para ello llamada MSSYS.SDO_GEOMETRY, que es usada para almacenar la geometría (punto, línea o polígono). El primer paso para almacenar los archivos es generar un esquema del archivo GML, simplifican el archivo GML mediante un Schema Tree Generation Algorithmy que determina los tipos de nodos y sus atributos. El paso siguiente es identificar las relaciones entre los elementos y/o atributos de un archivo GML con las tablas y columnas de una Geodatabase (véase Tabla 1). Al aplicar el algoritmo GMLSMA se obtiene el esquema de la base de datos espacial (Véase Tabla 2) [13]. La arquitectura propuesta por los autores permite identificar la estructura de la tabla pero no permite identificar el modelo entidad-relación de la base de datos espacial en Oracle.

Tabla 1. Relación entre los tipos de geometrías de GML y Oracle Spatial [13]

\begin{tabular}{|l|l|}
\hline \multicolumn{2}{|c|}{ Tipos de Geometrías } \\
\hline GML & $\begin{array}{l}\text { Oracle } \\
\text { (SDO_GTYPE) }\end{array}$ \\
\hline PointType & POINT \\
\hline LineStringType & LINE/CURVE \\
\hline PolygonType/BoxType & POLYGON \\
\hline
\end{tabular}




\begin{tabular}{|l|l|}
\hline \multicolumn{2}{|c|}{ Tipos de Geometrías } \\
\hline MultiPointType & MULTIPOINT \\
\hline MultiLineStringType & $\begin{array}{l}\text { MULTILINE/MULTICURV } \\
\text { E }\end{array}$ \\
\hline $\begin{array}{l}\text { MultiPolygonType/Line } \\
\text { arRingType }\end{array}$ & MULTIPOLYGON \\
\hline
\end{tabular}

Tabla 2. Estructura de la tabla en Spatial Database [13]

MAP table

\begin{tabular}{|l|l|}
\hline map.layer.featuremember.ID & Integer \\
\hline feature table & \\
\hline ID & Integer \\
\hline Feature.parentid & Integer \\
\hline $\begin{array}{l}\text { Feature.GeometryProperty.multipoi } \\
\text { nt }\end{array}$ & Multipoint \\
\hline Feature.Geometryproperty.point & Point \\
\hline $\begin{array}{l}\text { Feature.simpleproperty.floatpropert } \\
\text { y.area }\end{array}$ & Float \\
\hline $\begin{array}{l}\text { Feature.simpleproperty.stringproper } \\
\text { ty.system }\end{array}$ & String \\
\hline $\begin{array}{l}\text { Feature.simpleproperty.stringproper } \\
\text { ty.name }\end{array}$ & String \\
\hline
\end{tabular}

Gandhi et ál. (2007) plantean la necesidad de desarrollar modelos de datos en 3D para representar datos volumétricos. Por lo que describen tres modelos: redes espacio temporales, objetos en movimiento y lenguajes de marca. El modelo de redes espacio temporales considera el tiempo dependiente de los parámetros y topologías de la red, por ejemplo, en redes de transporte el tiempo de viaje en segmentos viales son dependiente del día y de cada intervalo. El modelo de objetos en movimiento se considera una base de datos espacio-temporal donde los objetos espaciales cambian su posición y extensión en un periodo de tiempo, por ejemplo, el movimiento de taxis, la trayectoria de un huracán y el perfil geográfico de criminales seriales. El objetivo del lenguaje de marca, como GML, es proveer un estándar para el lenguaje de modelado y formatos de intercambio de datos geográficos. Las entidades asociadas con datos espaciales como geometría, sistemas de coordenadas y atributos, entre otros, se pueden representar en un archivo GML (véase Figura 9) [14]. Los autores presentan el modelo entidad relación para representar los datos espaciales comunes, pero no muestran el diseño del modelo de gestión de datos geográficos para almacenar los archivos de lenguaje marcado.

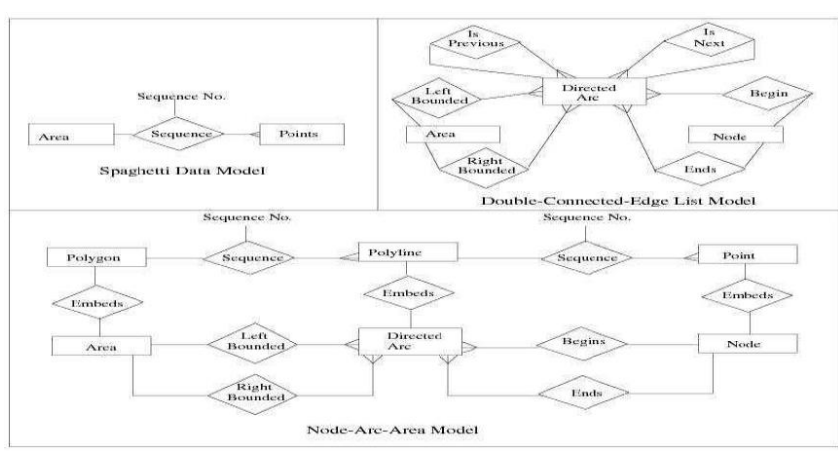

Figura 9. Diagrama entidad relación para representar datos espaciales comunes
García et ál. (2009) describen la implementación de una base de datos histórica en un SIG para entono Web. La base de datos permite consultas y análisis de datos espacio-temporales con una representación dinámica de los resultados. El principal objetivo de la base de datos es descubrir patrones de comportamiento y fenómenos dinámicos que esclarezcan 0 evidencien fenómenos históricos representados en el comercio de esclavos durante los siglos XVI al XIX. La estructura de la base de datos se basa en una hoja de Ms Excel, que se utilizó como punto de partida para generar los casos de uso y el diseño del modelo de clases. En la Figura 10 se muestra un fragmento del modelo de la base de datos que se generó después de la normalización de la hoja de Ms Excel [15]. El modelo presentado muestra el modelo conceptual en UML, los casos de uso y el diagrama de clases para realizar un prototipo de la interfaz de usuario en entorno Web, pero no se explica el diseño de modelo conceptual de la base de datos espacial ni la forma de almacenar los cambios en el tiempo de la información espacial.

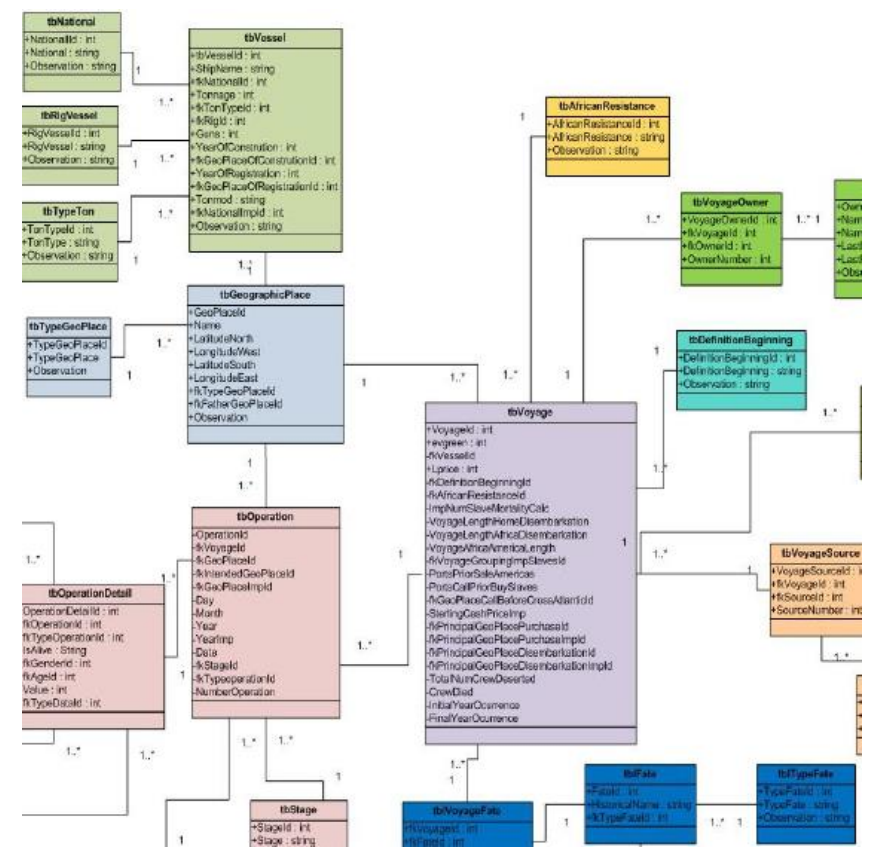

Figura 10. Fragmento del modelo conceptual de la base de datos en UML [15]

Deng y Zheng (2010) presentan un método para diseñar y construir una base de datos espacial sobre Temporal Geographic Information System (TGIS). TGIS es una tecnología desarrollada para trabajar con Sistemas de Información Geográfica que modela fenómenos geográficos con cambios en el tiempo. Los autores se basan en modelos SIG espacio-temporales utilizando objetos para diseñar la BDE y para establecer una relación entre ArcSDE y Oracle. Existen tres niveles en los modelos espacio-temporales: modelo conceptual espacio-temporal, modelo lógico de datos espaciales y modelo físico de datos espaciales. En la Figura 11 se observa el modelo entidad relación acorde con las reglas de asignación, utilizando UML. La base de datos desarrollada tiene las siguientes 
ventajas: habilidad para reflejar los estados del dato en tiempo real, almacenamiento de datos históricos con actualización de cambios, gestión visual de los estados históricos, el sistema es flexible y extensible. La desventaja de la base de datos es que no considera relaciones topológicas entre los objetos para reducir el tiempo de carga [16]. La base de datos se basa principalmente en el versionamiento de la información (espacial y no espacial). El versionamiento requiere crear una nueva versión para cada cambio de estado o de un objeto espacial. El método tiene alto costo de almacenamiento, al requerir guardar información en cada cambio del objeto espacial en el tiempo y no se observa en el modelo la forma de incluir formatos espacio-temporales.

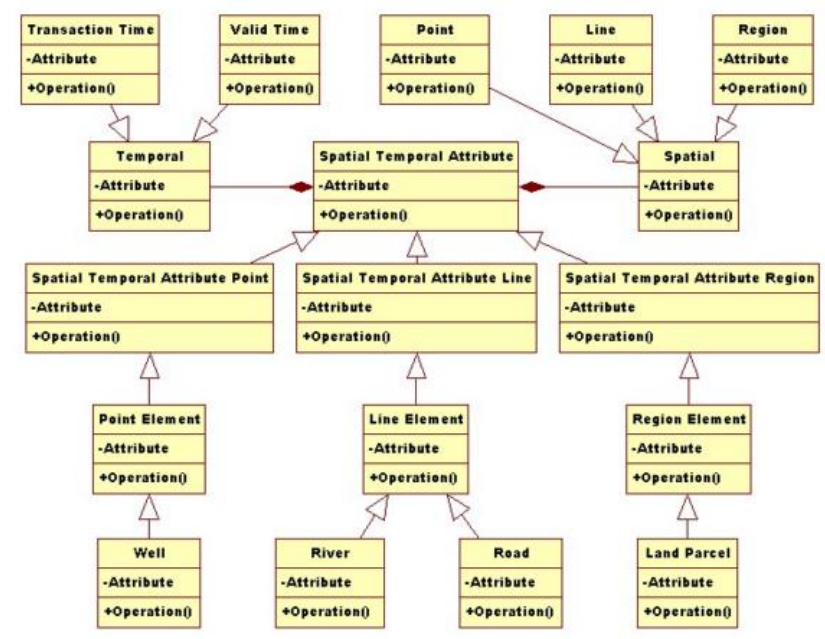

Figura 11. Diagrama de clases

Peuquet y Duan (2014) presentan un "Modelo de Datos Espacio Temporal Basado en Eventos" (siglas en inglés ESTDM) basado en la variable "Tiempo". El modelo cuenta con tres formas de recuperar la información: (1) cambio de un momento dado, (2) cambio en un intervalo temporal determinado; y (3) cálculo de la superficie total durante un intervalo temporal. ESTDM es una forma de almacenamiento que asocia cambios específicos con el tiempo, generando una lista de eventos. Una desventaja de los modelos de almacenamiento con cambios está relacionado con la cantidad de área relacionada con la ubicación de los trillizos ( $x, y, v)$, es decir, la cantidad total de posiciones discretas que cambian en el tiempo. ESTDM reduce este inconveniente, donde primero agrupa la información por la misma posición de las coordenadas $(x, y)$ los datos con valores específicos para $\checkmark$ se almacenan en un subestructura llamada "Component". Lo anterior se resumen en, cada valor se almacena una vez por cada evento para cada posición $(\mathrm{x}, \mathrm{y})$ y una estructura "Component" se almacena para cada celda que cambia de posición (véase Figura 12) [17]. El modelo describe el almacenamiento teniendo en cuenta el tiempo, realiza distinción entre cuándo un estado o condición es correcto o valido para el mundo real y cuándo es almacenado en la base de datos, pero no describe la estructura de la base de datos espacial que conecte la información no espacial con la espacial y los cambios en el tiempo.

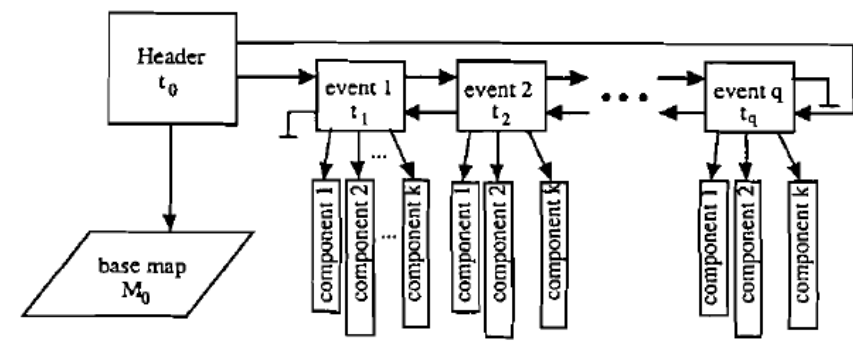

Figura 12. Estructura y elementos del ESTDM [17]

ArcSDE es una tecnología de ESRI ${ }^{\mathrm{TM}}$ para acceder $\mathrm{y}$ administrar datos geoespaciales dentro de las bases de datos relacionales. La tecnología de ArcSDE admite la lectura y la escritura de varios estándares como Open Geospatial Consortium, Inc. (OGC) para entidades simples, el estándar de la Organización Internacional para la Estandarización (ISO) para tipos espaciales y las bases de datos relacionales de Oracle, PostGIS y Microsoft SQL Server, entre otros [1]. ArcSDE permite multiusuarios para la edición y consulta de información geográfica, soporte al versionamiento de la base de datos espacial para la copia y carga de datos mediante check-out y check-in y archivos históricos. ArcGIS permite transferir datos entre bases de datos espaciales utilizando XML. Con el formato XML de ArcGIS se puede importar y exportar los elementos y datos de una base de datos espacial con dominios, roles, capas temática y topologías. En la Figura 13 se observa el modelo del sistema de tablas de ArcSDE para las bases de datos espaciales compatibles con ArcGIS (Oracle, PostgreSQL, DB2, entre otras). El modelo ArcSDE XML propuesto permite compartir información espacial y no espacial con otros motores de bases de datos espaciales, pero no se evidencia la forma de almacenar la información que contiene un archivo de datos espacio-temporal.

\section{SOLUCIÓN PROPUESTA}

La solución que se presenta en esta sección mejora y facilita el proceso de interoperabilidad entre formatos de datos espacio-temporales, específicamente con los formatos XML, KML y GML. Las mejoras se realizan en términos del modelo de base de datos propuesto para la incorporación de la información de los formatos de datos espacio-temporales. Cada formato de datos espacio-temporales presenta información común que se asocia con: nombre del archivo, nombre del elemento espacial, geometría espacial, coordenadas geográfica, identificador, tiempo de toma de los datos, unidades y valores (véase Tabla 3) [2]. 


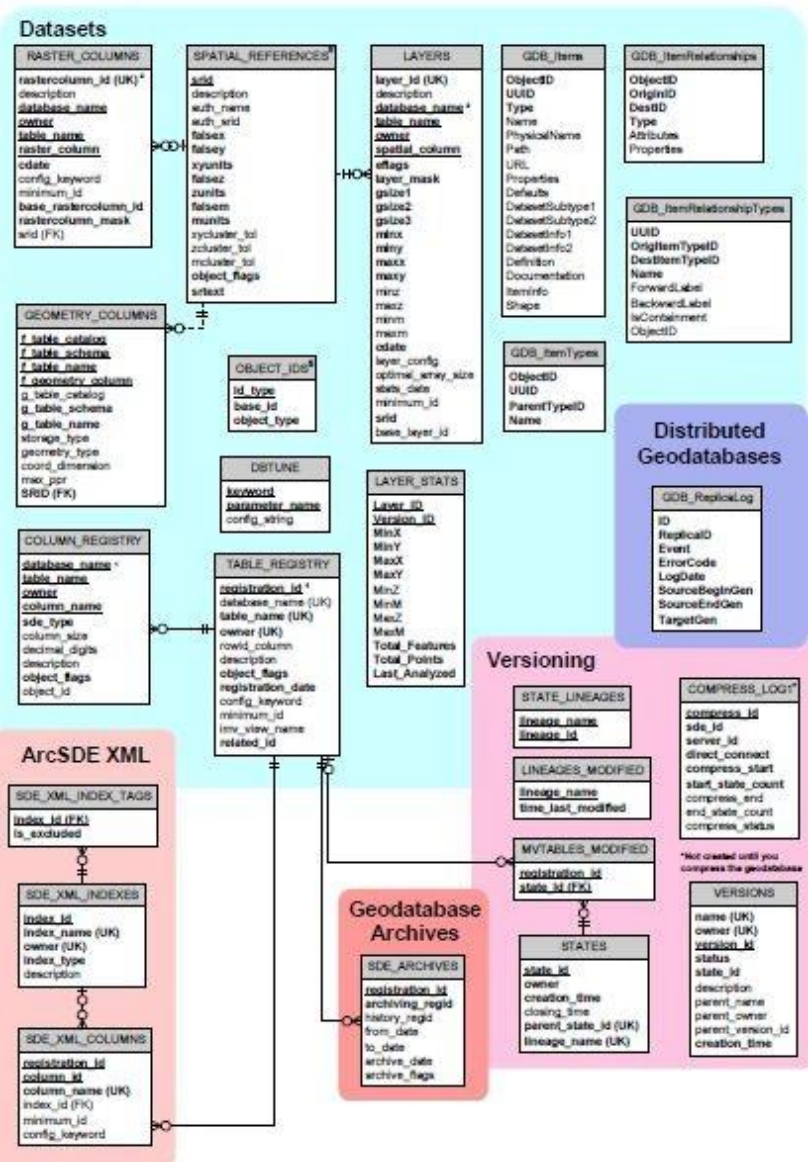

Figura 13. ArcSDE Geodatabase System Tables

Tabla 3. Elementos comunes de los formatos de datos espacio-temporales [2] (1/3)

\begin{tabular}{|l|l|}
\hline \multicolumn{2}{|l|}{ Nombre del archivo } \\
\hline XML & Namespace \\
\hline GML & Name \\
\hline KML & Name \\
\hline Encabezado \\
\hline \multirow{4}{*}{ XML } & Header \\
\cline { 2 - 2 } & $\bullet$ Version \\
\cline { 2 - 2 } & $\bullet$ Encoding \\
\hline \multirow{4}{*}{ GML } & Header \\
\cline { 2 - 2 } & $\bullet$ Version \\
\cline { 2 - 2 } & $\bullet$ Encoding \\
\hline \multirow{4}{*}{ KML } & Header \\
\cline { 2 - 2 } & $\bullet$ Version \\
\cline { 2 - 2 } & $\bullet$ Encoding \\
\hline
\end{tabular}

Tabla 3. Elementos comunes de los formatos de datos espacio-temporales [2] (2/3)

Descripción de los Datos, los valores dependen del tipo de información descriptiva que requiera el usuario

\begin{tabular}{|l|l|}
\hline \multirow{3}{*}{ XML } & $\bullet$ Dataset \\
\cline { 2 - 3 } & $\bullet$ Observation \\
\cline { 2 - 2 } & $\bullet$ Type \\
\hline
\end{tabular}

\begin{tabular}{|c|c|}
\hline \multicolumn{2}{|c|}{$\begin{array}{l}\text { Descripción de los Datos, los valores dependen del tipo } \\
\text { de información descriptiva que requiera el usuario }\end{array}$} \\
\hline & - Description \\
\hline & - Source \\
\hline \multirow{7}{*}{ GML } & Namespace \\
\hline & - Namespace \\
\hline & - Shema versión \\
\hline & - ElementfromDefault \\
\hline & - Xmlns \\
\hline & - TargetNamespace \\
\hline & - $X s i$ \\
\hline \multirow{3}{*}{ KML } & Namespace \\
\hline & - Xmlns \\
\hline & - Xmlns:gx \\
\hline \multicolumn{2}{|r|}{ Elemento Geográfico } \\
\hline $\mathrm{XML}$ & Feature \\
\hline GML & Shema \\
\hline KML & Parameter \\
\hline \multicolumn{2}{|r|}{ Identificador de elemento geográfico } \\
\hline $\mathrm{XML}$ & Feature|FID \\
\hline GML & ShemalFID \\
\hline KML & Parameter|FID \\
\hline \multicolumn{2}{|r|}{ Nombre del elemento geográfico } \\
\hline $\mathrm{XML}$ & Feature|Name \\
\hline GML & ShemalComplextimelElement|Name \\
\hline KML & ParameterIName \\
\hline \multicolumn{2}{|c|}{$\begin{array}{l}\text { Propiedad geométrica, está representada por el tipo de } \\
\text { elemento geográfico, e.g. punto, línea, polígono, curvas, } \\
\text { superficie }\end{array}$} \\
\hline $\mathrm{XML}$ & FeaturelGeometryProperty \\
\hline GML & ShemalGeometryProperty \\
\hline KML & Parameter|GeometryProperty \\
\hline \multicolumn{2}{|r|}{ Coordenadas geográfica } \\
\hline \multirow{2}{*}{ XML } & Feature|Coordinates $\mid X$ \\
\hline & Feature|Coordinates $\mid Y$ \\
\hline \multirow{2}{*}{ GML } & ShemalCoordinates $\mid X$ \\
\hline & ShemalCoordinates $\mid Y$ \\
\hline \multirow{2}{*}{ KML } & Parameter|Coordinates $\mid X$ \\
\hline & Parameter|Coordinates $\mid Y$ \\
\hline
\end{tabular}

Tabla 4. Elementos comunes de los formatos de datos espacio-temporales [2] (3/3)

\begin{tabular}{|c|c|}
\hline \multicolumn{2}{|c|}{ Campo de asignación del tiempo de toma del dato } \\
\hline $\mathrm{XML}$ & Feature|Variable|Parameter|Data|Time \\
\hline \multirow{2}{*}{ GML } & ShemalComplextime|Element|DatalFecha \\
\hline & Shema|ComplextimelElement|DatalHora \\
\hline \multirow{2}{*}{ KML } & Parameter|DatalFecha \\
\hline & Parameter|DatalHora \\
\hline \multicolumn{2}{|c|}{ Campo con la información asociada } \\
\hline \multirow{5}{*}{$\mathrm{XML}$} & Feature|Variable|Parameter|Name \\
\hline & Feature|Variable|Parameter|Unit \\
\hline & Feature|Variable|Parameter|Nodata \\
\hline & $\begin{array}{l}\begin{array}{l}\text { Feature|Variable|Parameterlstatistics } \\
\text { (average or sum) }\end{array} \\
\end{array}$ \\
\hline & Feature|Variable|Parameter|Data|Value \\
\hline GML & $\begin{array}{l}\text { ShemalComplextime|Element|Datal"Nombre } \\
\text { del campo" }\end{array}$ \\
\hline
\end{tabular}




\begin{tabular}{|l|l|}
\hline \multirow{4}{*}{ KML } & $\begin{array}{l}\text { Se puede presentar que el existen varios } \\
\text { campos, se indica cada uno de ellos, e.g. } \\
\text { Temperatura, Oxigeno, pH, DBO5 con sus } \\
\text { respectivos valores. }\end{array}$ \\
\hline \multirow{3}{*}{\begin{tabular}{l} 
Parameter|Datal"Nombre del campo" \\
\cline { 2 - 3 } Se puede presentar que el existen varios \\
campos, se indica cada uno de ellos, e.g. \\
Temperatura, Oxigeno, pH, DBO5 con sus \\
respectivos valores.
\end{tabular}} \\
\cline { 2 - 2 } & $\begin{array}{l}\text { Parameter|DatalType (table, arraysimple or } \\
\text { arraycomplex) }\end{array}$ \\
\hline
\end{tabular}

Zapata y Durango (2013) utilizaron los Esquemas Preconceptuales para identificar los elementos comunes de los formatos de datos espacio-temporales [2]. En las Figuras 14, 15 y 16 se presentan los Esquemas Preconceptuales de los formatos de datos espacio-temporales de los formatos XML, GML y KML donde se puede apreciar los principales elementos.

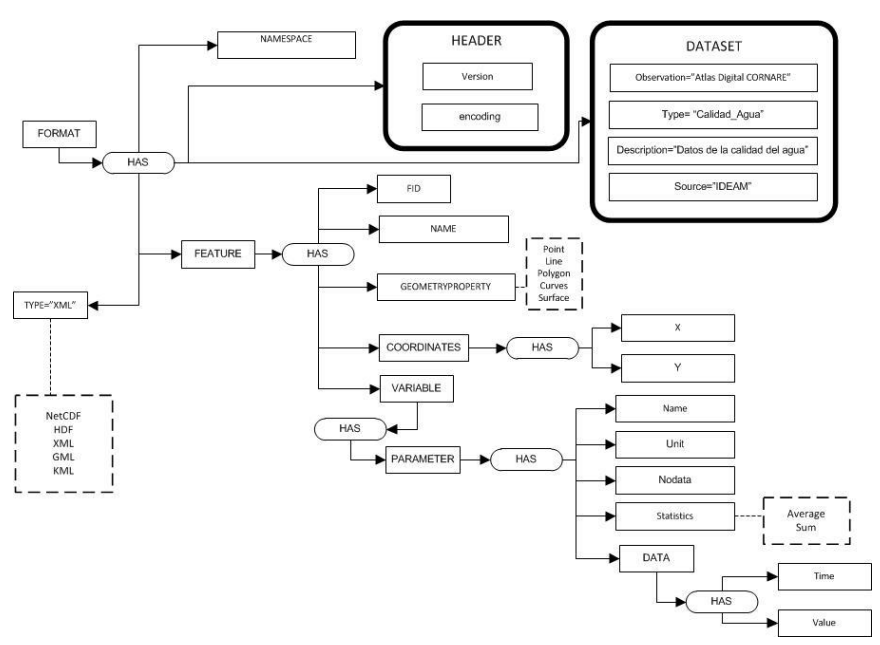

Figura 14. Elementos principales del formato XML, utilizando Esquemas Preconceptuales [2]

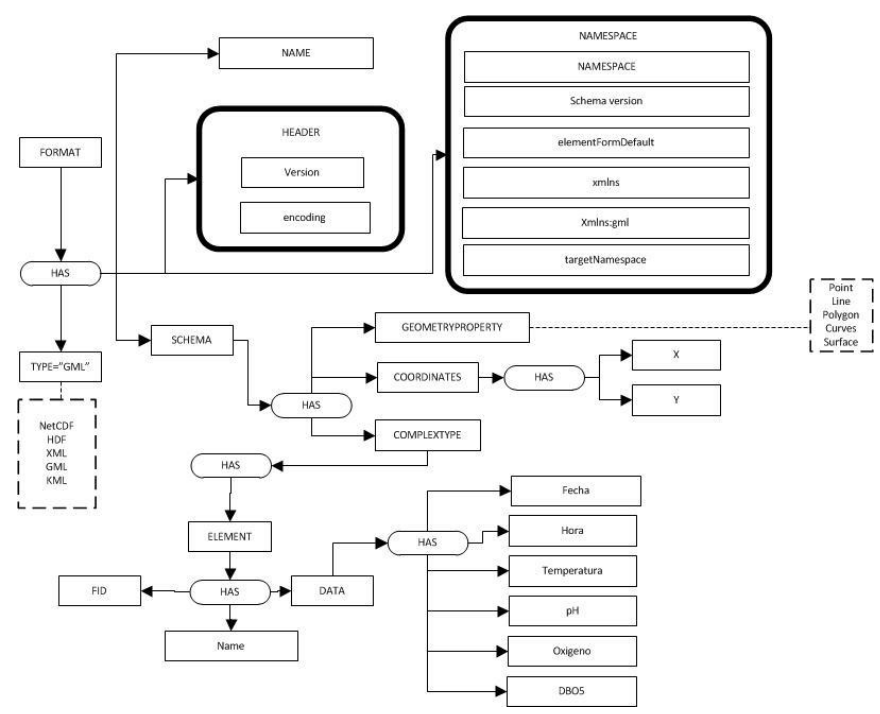

Figura 15. Elementos principales del formato GML, utilizando Esquemas Preconceptuales [2]

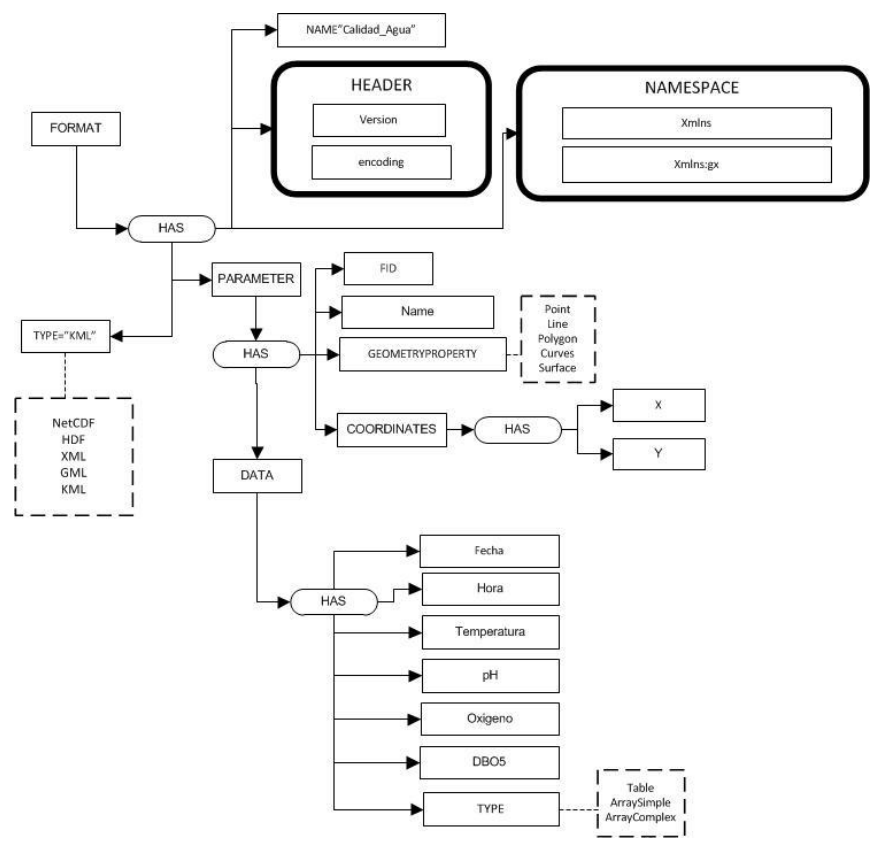

Figura 16. Elementos principales del formato KML, utilizando Esquemas Preconceptuales [2]

En las Figuras 17, 18 y 19 se presentan los modelos entidad-relación propuestos para los formatos de datos espacio-temporales XML, GML y KML.

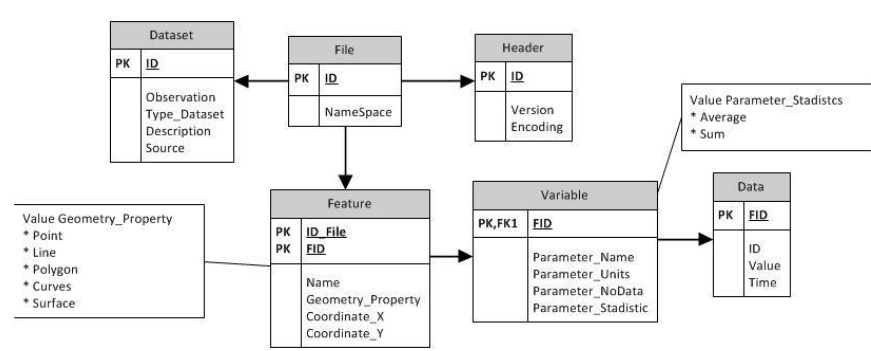

Figura 17. Modelo de datos relacional para el formato XML, utilizando Microsoft Visio 2010. Construcción propia

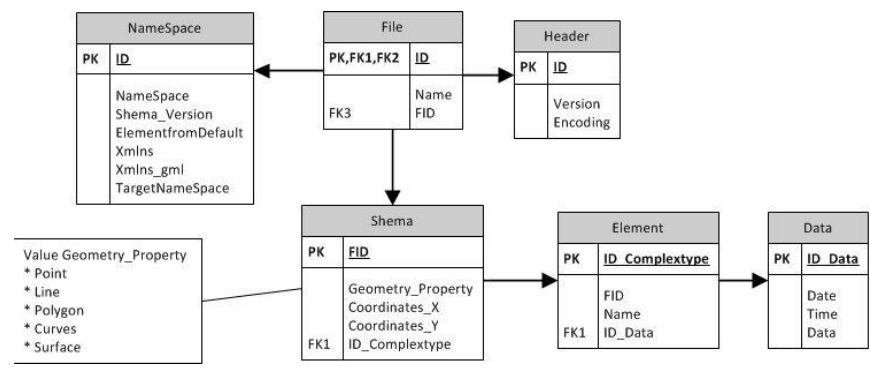

Figura 18. Modelo de datos relacional para el formato GML, utilizando Microsoft Visio 2010. Construcción propia 


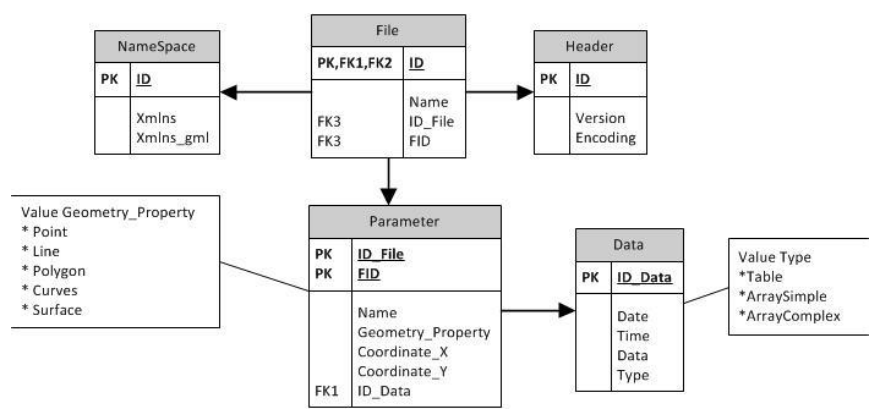

Figura 19. Modelo de datos relacional para el formato KML, utilizando Microsoft Visio 2010. Construcción propia

\section{CONCLUSIONES Y TRABAJOS FUTUROS}

\subsection{Conclusiones}

El uso de los Sistemas de Información Geográfica se encuentra en crecimiento en los últimos años. Los formatos de datos espacio-temporales ayudan al intercambio de grandes cantidades de información y apoyan el almacenamiento de información temporal. Por lo anterior, en este proyecto se abordan tres formatos de datos espacio-temporales para almacenamiento de datos vector: XML, GML y KML.

Para demostrar que los datos de los formatos espaciotemporales, se pueden almacenar en un modelo de bases de datos, se modelan los elementos principales de los formatos XML, GML y KML. Para realizar el modelo se utilizó la herramienta Microsoft Visio 2010, que permite generar el modelo geográfico y de la base de datos al permitir exportar a Oracle.

Como resultado se observa que los modelos de los tres formatos tienen elementos similares que permiten la estandarización del modelo de bases de datos. Lo anterior constituye una herramienta de apoyo para el almacenamiento de información espacio-temporal en modelos de gestión de información.

\subsection{Trabajo futuro}

En este trabajo se abordan tres formatos de datos espacio-temporales, por lo que se recomienda incorporar otros dos formatos de almacenamiento de los datos espaciales NetCDF y HDF para datos ráster. Además generar un script que permita importar los formatos espacio-temporales al modelo de gestión de datos geográficos en Oracle.

\section{REFERENCIAS}

[1] "ArcGIS Resources Center," 2014. [Online]. Available:

http://help.arcgis.com/es/arcgisdesktop/10.0/help/ind ex.html\#/na/005s00000038000000/.
[2] C. M. Zapata J. and C. E. Durango V., "Representación del conocimiento en datos espaciotemporales para Sigs: un enfoque basado en esquemas preconceptuales," Rev. Fac. Ing. USBMed, vol. 4, no. 1, pp. 47-55, 2013.

[3] B. Martínez, M. Torres, and M. Moreno, "Bases de Datos Espacio-Temporales 1," Instituto Politécnico Nacional, México, D.F., 2008.

[4] C. M. Zapata J., G. G. G. L., and S. Londoño V., "Esquemas Preconceptuales ejecutables," Rev. Av. en Sist. e Informática, vol. 8, no. 1, pp. 15-23, 2011.

[5] D. P. Ames, K. Asch, N. Bartelme, M. Becker, W. Kresse, and D. M. Danko, Springer handbook of Geographic Information. 2012, p. 1132.

[6] "Open Geospatial Consortium," Open Geospatial Consortium. OpenGIS $\AA^{8}$ and OGC $\AA$, 2014. [Online]. Available: http://www.opengeospatial.org/.

[7] "W3C," W3C Standards, 2014. [Online]. Available: http://www.w3.org.

[8] Y. Du, C. Yu, and J. Liu, "A Study of GIS Development Based on KML and Google Earth," in 2009 Fifth International Joint Conference on INC, IMS and IDC, 2009, pp. 1581-1585.

[9] M. Breunig and S. Zlatanova, "3D geo-database research: Retrospective and future directions," Comput. Geosci., vol. 37, no. 7, pp. 791-803, Jul. 2011.

[10] R. Nandal, "Spatio-Temporal Database and Its Models: A Review," IOSR J. Comput. Eng., vol. 11, no. 2, pp. 91-100, 2013.

[11] M. Erwig, R. Hartmut, G. Markus, S. Michalis, and D.- Hagen, "Abstract and Discrete Modeling of Spatio-Temporal Data Types," in GIS '98 Proceedings of the 6th ACM international symposium on Advances in geographic information systems, 1998, pp. 131-136.

[12] B. Li and G. Cai, "A general object-oriented spatial temporal data model," in Symposium on Geospatial Theory, 2002.

[13] Y. Li, J. Li, and S. Zhou, GML Storage: A Spatial Database Approach. Springer-Verlag Berlin Heidelberg 2004, 2004, pp. 55-66.

[14] V. Gandhi, J. Kang, and S. Shekhar, "Spatial Databases," Minneapolis, 2007.

[15] M. J. G. Rodríguez, A. U. Zambrana, and M. A. Bernabé, "Diseño de herramientas de análisis espacio-temporales para el estudio de bases de datos históricas," in GTIDEE 2009, 2009, p. 16.

[16] J. Deng and X. Zheng, "Design and Construction of Spatial Database for Internal Elements of Urban Based on TGIS," in Geoinformatics, 2010 18th International Conference on, 2010, no. 40571119, pp. 1-6.

[17]D. J. Peuquet and N. Duan, "An event-based spatiotemporal data model (ESTDM) for temporal analysis of geographical data," Int. J. Geogr. Inf. Syst., vol. 9, no. 1, pp. 7-24, Jan. 1995. 